Stud. Univ. Babeş-Bolyai Math. 66(2021), No. 3, 457-470

DOI: 10.24193/subbmath.2021.3.04

\title{
Differential subordination for Janowski functions with positive real part
}

\author{
Swati Anand, Sushil Kumar and V. Ravichandran
}

\begin{abstract}
Theory of differential subordination provides techniques to reduce differential subordination problems into verifying some simple algebraic condition called admissibility condition. We exploit the first order differential subordination theory to get several sufficient conditions for function satisfying several differential subordinations to be a Janowski function with positive real part. As applications, we obtain sufficient conditions for normalized analytic functions to be Janowski starlike functions.
\end{abstract}

Mathematics Subject Classification (2010): 30C45.

Keywords: Subordination, univalent functions, Carathéodory functions, starlike functions, Janowski function, admissible function.

\section{Motivation}

The class of all analytic functions defined on the unit disk $\mathbb{D}:=\{z \in \mathbb{C}:|z|<1\}$ that fixes the origin and has derivative 1 at the origin is denoted by $\mathcal{A}$. An analytic function $p$ is subordinate to the analytic function $q$, written $p \prec q$, if $p=q \circ \omega$ for some analytic function $\omega: \mathbb{D} \rightarrow \mathbb{D}$ with $\omega(0)=0$. If the function $q$ is univalent in $\mathbb{D}$, then $p \prec q$ if and only if $p(0)=q(0)$ and $p(\mathbb{D}) \subseteq q(\mathbb{D})$. The class $\mathcal{P}$ consists of Carathéodory functions $p: \mathbb{D} \rightarrow \mathbb{C}$ of the form $p(z)=1+c_{1} z+c_{2} z+\cdots$ that maps the unit disk $\mathbb{D}$ into a region on the right half plane. For arbitrary fixed numbers $A$ and $B$ satisfying $-1 \leq B<A \leq 1$, denote by $\mathcal{P}[A, B]$ the class of analytic functions $p \in \mathcal{P}$ satisfy the subordination $p(z) \prec(1+A z) /(1+B z)$. We call the functions in $\mathcal{P}[A, B]$ as Janowski functions with positive real part. The class $\mathcal{S}^{*}[A, B]$ consists of functions $f \in \mathcal{A}$ such that $z f^{\prime}(z) / f(z) \in \mathcal{P}[A, B]$ for $z \in \mathbb{D}$. The functions in the class $\mathcal{S}^{*}[A, B]$ are called the Janowski starlike functions, introduced by Janowski [12]. In particular, $S^{*}[1-2 \alpha,-1]=\mathcal{S}^{*}(\alpha)$ is the class of starlike functions of order $\alpha$, see $[11,23]$.

Nunokawa [21] proved that if $1+z p^{\prime}(z) \in \mathcal{P}[1,0]$, then $p \in \mathcal{P}[1,0]$. In 2007, Ali et al. [3] determined the conditions on $\beta$ and numbers $A, B, D, E \in[-1,1]$ so 
that $p \in \mathcal{P}[A, B]$ whenever $1+\beta z p^{\prime}(z)$ or $1+\beta z p^{\prime}(z) / p(z)$ or $1+\beta z p^{\prime}(z) / p^{2}(z)$ is in the class $\mathcal{P}[D, E]$. In 2018, authors [17] obtained the sharp lower bound on $\beta$ so that the function $p(z)$ is subordinate to the functions $e^{z}$ and $(1+A z) /(1+B z)$ whenever $1+\beta z p^{\prime}(z) / p^{j}(z),(j=0,1,2)$ is subordinate to the functions with positive real part like $\sqrt{1+z},(1+A z) /(1+B z)$. Recently, Ahuja et al. [1] computed sharp estimates for $\beta$ so that a Carathéodory function is subordinate to a starlike function with positive real part whenever $1+\beta z p^{\prime}(z) / p^{j}(z),(j=0,1,2)$ is subordinate to lemniscate starlike function. For more details, see $[6,8,21,25,26]$. Motivated by work done in $[1,3,5,7,9,10,17]$, by using admissibility condition technique, a condition on $\beta$ is established so that $p \in \mathcal{P}[A, B]$ when $1+\beta p^{\prime}(z) / p^{k}(z)$ with $k \in$ $\mathbb{N} \cup\{0\}, p(z)+\beta z p^{\prime}(z) / p^{2}(z), 1+\beta\left(z p^{\prime}(z)\right)^{2} / p^{k}(z)$ and $1 / p(z)-\beta z p^{\prime}(z) / p^{k}(z)$ for $k \in \mathbb{N} \cup\{0\}$ are in the class $\mathcal{P}[D, E]$. We compute a condition on $\alpha$ and $\beta$ for $p \in$ $\mathcal{P}[A, B]$ whenever $(1-\alpha) p(z)+\alpha p^{2}(z)+\beta z p^{\prime}(z) / p^{k}(z) \in \mathcal{P}[D, E]$ for $k=0,1$ as well. Additionally, a condition on $\beta$ and $\gamma$ is determined in a Briot-Bouquet differential type subordination relation: $p(z)+z p^{\prime}(z) /(\beta p(z)+\gamma)^{2} \in \mathcal{P}[D, E]$ implies $p \in \mathcal{P}[A, B]$. As an application, we obtained some sufficient conditions for a normalized analytic function $f$ in $\mathcal{S}^{*}[A, B]$. Kanas [14] described the admissibility condition for the function to map $\mathbb{D}$ on to region bounded by parabola and hyperbola. We prove our result by using the corresponding admissibility conditions for the Janowski functions with positive real part.

\section{Janowski functions}

Let $\psi(r, s, t ; z): \mathbb{C}^{3} \times \mathbb{D} \rightarrow \mathbb{C}$ be a function and let $h$ be univalent in $\mathbb{D}$. An analytic function $p$ satisfying the second-order differential subordination

$$
\psi\left(p(z), z p^{\prime}(z), z^{2} p^{\prime \prime}(z) ; z\right) \prec h(z),
$$

is known as its solution. The univalent function $q$ is a dominant of the solutions of the differential subordination (2.1) if $p \prec q$ for all $p$ satisfying (2.1). A dominant $\tilde{q}$ which satisfies $\tilde{q} \prec q$ for all dominant $q$ of (2.1) is known as best dominant of (2.1) and it is unique up to a rotation. Let $\mathcal{Q}$ be the class consisting of all analytic and injective functions $q$ on $\overline{\mathbb{D}} \backslash \mathbf{E}(q)$, where $\mathbf{E}(q)=\left\{\xi \in \partial \mathbb{D}: \lim _{z \rightarrow \xi} q(z)=\infty\right\}$ such that $q^{\prime}(\xi) \neq 0$ for $\xi \in \overline{\mathbb{D}} \backslash \mathbf{E}(q)$. Let $\Omega$ be a set in $\mathbb{C}, q \in \mathcal{Q}$ and $n$ be a positive integer. The class $\Psi_{n}[\Omega, q]$ of admissible functions $\psi: \mathbb{C}^{3} \times \mathbb{D} \rightarrow \mathbb{C}$ that satisfy the admissibility condition:

$$
\psi(r, s, t ; z) \notin \Omega
$$

whenever

$$
r=q(\xi), s=m \xi q^{\prime}(\xi) \text { and } \operatorname{Re}\left(\frac{t}{s}+1\right) \geq m \operatorname{Re}\left(\frac{\xi q^{\prime \prime}(\xi)}{q^{\prime}(\xi)}+1\right)
$$

for $z \in \mathbb{D}, \xi \in \overline{\mathbb{D}} \backslash \mathbf{E}(q)$ and $m \geq n \geq 1$. In particular, let $\Psi_{1}[\Omega, q]=\Psi[\Omega, q]$. For more details, see $[4,13,15,16,19,24]$. For this class $\Psi_{n}[\Omega, q]$, the following result is well-known. 
Theorem 2.1. [20, Theorem 2.3b, p. 28] Let the function $\psi \in \Psi_{n}[\Omega, q]$ with $q(0)=a$. If the function $p \in \mathcal{H}[a, n]$ satisfies

$$
\psi\left(p(z), z p^{\prime}(z), z^{2} p^{\prime \prime}(z) ; z\right) \in \Omega
$$

then $p(z) \prec q(z)$.

We begin by describing the class of admissible function $\Psi_{n}[\Omega, q]$ when $q: \mathbb{D} \rightarrow \mathbb{C}$ is the function given by $q(z)=(1+A z) /(1+B z)$ where $-1 \leq B<A \leq 1$. Note that $q(0)=1$ and $E(q) \subset\{-1\}$. Clearly, the function $q$ is univalent in $\overline{\mathbb{D}} \backslash \mathbf{E}(q)$. Therefore $q \in \mathcal{Q}$ and the domain $q(\mathbb{D})$ is

$$
\Delta=q(\mathbb{D})=\left\{w \in \mathbb{C}:\left|\frac{w-1}{A-B w}\right|<1\right\} .
$$

For $\varsigma=e^{i \theta}$ and $0<\theta<2 \pi$, we have

$$
q(\varsigma)=\frac{1+A e^{i \theta}}{1+B e^{i \theta}}, q^{\prime}(\varsigma)=\frac{A-B}{\left(1+B e^{i \theta}\right)^{2}} \text { and } q^{\prime \prime}(\varsigma)=\frac{-2 B(A-B)}{\left(1+B e^{i \theta}\right)^{3}} .
$$

and a simple calculation yields

$$
\operatorname{Re}\left(\frac{\varsigma q^{\prime \prime}(\varsigma)}{q^{\prime}(\varsigma)}+1\right)=\frac{1-B^{2}}{1+B^{2}+2 B \cos \theta} .
$$

Thus we get the following condition of admissibility: $\psi(r, s, t ; z) \notin \Omega$ whenever $(r, s, t ; z) \in \operatorname{dom} \psi$ and

$$
r=\frac{1+A e^{i \theta}}{1+B e^{i \theta}}, s=\frac{m(A-B) e^{i \theta}}{\left(1+B e^{i \theta}\right)^{2}} \text { and } \operatorname{Re}\left(\frac{t}{s}+1\right) \geq \frac{\left(1-B^{2}\right) m}{1+B^{2}+2 B \cos \theta}
$$

where $0<\theta<2 \pi$ and $m \geq n \geq 1$ and the class of all such functions $\psi$ satisfying the admissibility condition is denoted by $\Psi(\Omega ; A, B)$.

When $q(z)=(1+A z) /(1+B z)$, Theorem 2.1 specializes to the following first order differential subordination result:

Theorem 2.2. Let $p \in \mathcal{H}[1, n]$ with $n \in \mathbb{N}$. Let $\Omega$ be a subset of $\mathbb{C}$ and $\psi: \mathbb{C}^{2} \times \mathbb{D} \rightarrow \mathbb{C}$ with domain $D$ satisfy $\psi(r, s ; z) \notin \Omega$ for all $z \in \mathbb{D}$, where $r$ and $s$ are given by $(2.4)$. If $\left(p(z), z p^{\prime}(z) ; z\right) \in D$ and $\psi\left(p(z), z p^{\prime}(z) ; z\right) \in \Omega$ for $z \in \mathbb{D}$, then $p \in \mathcal{P}[A, B]$.

We investigate functions that naturally arise in the investigation of univalent functions to be admissible. In the first result, we show that $\psi(r, s ; z)=1+\beta s / r^{k}$ is an admissible function.

Theorem 2.3. Let $\beta \neq 0,-1 \leq B<A \leq 1$ and $-1 \leq E<D \leq 1$ satisfy the condition

(i) $|\beta|(A-B) \geq(D-E)(1+|A|)^{k}(1+|B|)^{2-k}+|E \beta(A-B)| ;(k=0,1,2)$ or

(ii) $|\beta|(A-B)(1-|B|)^{k-2} \geq(D-E)(1+|A|)^{k}+|E \beta(A-B)|(1+|B|)^{k-2} ;(k>2)$.

If $p$ is analytic in $\mathbb{D}$ and

$$
1+\beta \frac{z p^{\prime}(z)}{p^{k}(z)} \in \mathcal{P}[D, E] ; \quad k \in \mathbb{N} \cup\{0\},
$$

then $p \in \mathcal{P}[A, B]$. 
Proof. Let $\Omega=\{w \in \mathbb{C}:|(w-1) /(D-E w)|<1\}$.

The function $\psi:(\mathbb{C} \backslash\{0\}) \times \mathbb{C} \times \mathbb{D} \rightarrow \mathbb{C}$ is defined as

$$
\psi(r, s ; z)=1+\beta \frac{s}{r^{k}}
$$

where $k$ is a non-negative integer. Using the values of $r, s$ from (2.4), we have

$$
\psi(r, s ; z)=1+\beta \frac{m(A-B) e^{i \theta}\left(1+B e^{i \theta}\right)^{k}}{\left(1+A e^{i \theta}\right)^{k}\left(1+B e^{i \theta}\right)^{2}} .
$$

By making use of Theorem 2.2, the desired subordination is showed if we prove $\psi \in$ $\Psi[\Omega ; A, B]$. For this purpose, set

$$
\chi(w, D, E)=\left|\frac{w-1}{D-E w}\right| .
$$

(i) When $k=0,1,2$. A simple calculation gives

$$
\begin{aligned}
|\chi(\psi(r, s ; z), D, E)| & =\left|\frac{\beta m(A-B) e^{i \theta}}{(D-E)\left(1+A e^{i \theta}\right)^{k}\left(1+B e^{i \theta}\right)^{2-k}-E \beta m e^{i \theta}(A-B)}\right| \\
& \geq \frac{|\beta| m(A-B)}{(D-E)\left|\left(1+A e^{i \theta}\right)^{k}\right|\left|\left(1+B e^{i \theta}\right)^{2-k}\right|+|E \beta m(A-B)|} \\
& \geq \frac{|\beta| m(A-B)}{(D-E)(1+|A|)^{k}(1+|B|)^{2-k}+m|E \beta(A-B)|} \\
& =: \phi(m) .
\end{aligned}
$$

Observe that the function $\phi(m)$ is an increasing function for $m \geq 1$ by first derivative test. Hence the minimum value of $\phi(m)$ occurs at $m=1$. Thus, the last inequality becomes

if the inequality

$$
|\chi(\psi(r, s ; z), D, E)| \geq \phi(1) \geq 1
$$

$$
|\beta|(A-B) \geq(D-E)(1+|A|)^{k}(1+|B|)^{2-k}+|E \beta(A-B)|
$$

holds. Therefore, $\psi(r, s ; z) \notin \Omega$ which implies $\psi \in \Psi(\Omega ; A, B)$ and we get the desired $p \prec q$.

(ii) When $k>2$, we note that

$$
\begin{aligned}
|\chi(\psi(r, s ; z), D, E)| & =\left|\frac{\beta m e^{i \theta}(A-B)\left(1+B e^{i \theta}\right)^{k-2}}{(D-E)\left(1+A e^{i \theta}\right)^{k}-E \beta m e^{i \theta}(A-B)\left(1+B e^{i \theta}\right)^{k-2}}\right| \\
& \geq \frac{|\beta| m(A-B)(1-|B|)^{k-2}}{(D-E)(1+|A|)^{k}+m|E \beta(A-B)|(1+|B|)^{k-2}} \\
& =: \phi(m) .
\end{aligned}
$$

As previous case, note that $\phi(m) \geq \phi(1)$. Hence the last inequality is written as

$$
|\chi(\psi(r, s ; z), D, E)| \geq 1
$$

provided

$$
|\beta|(A-B)(1-|B|)^{k-2} \geq(D-E)(1+|A|)^{k}+|E \beta(A-B)|(1+|B|)^{k-2} .
$$

Therefore, we get $p \prec q$. 
Remark 2.4. When $k=0$ and 1 , Theorem 2.3 reduces to [3, Lemma 2.1, p. 2] and [3, Lemma 2.10 , p. 6] respectively. When $k=2$ and $\beta=1$, Theorem 2.3 simplifies to [3, Lemma 2.6, p. 5].

For a positive integer $k$, next theorem gives a conditions on $\beta$ so that the differential subordination

$$
1+\beta\left(z p^{\prime}(z)\right)^{2} / p^{k}(z) \in \mathcal{P}[D, E]
$$

implies $p \in \mathcal{P}[A, B]$.

Theorem 2.5. Suppose $k$ is a non-negative integer, $\beta \neq 0,-1 \leq B<A \leq 1$ and $-1 \leq E<0<D \leq 1$ satisfy either

(i) for $0 \leq k<4$,

$$
|\beta|(A-B)^{2} \geq(D-E)(1+|A|)^{k}(1+|B|)^{4-k}+\left|E \beta(A-B)^{2}\right|,
$$

or

(ii) for $k \geq 4$,

$$
|\beta|(A-B)^{2}(1-|B|)^{k-4} \geq(D-E)(1+|A|)^{k}+\left|E \beta(A-B)^{2}\right|(1+|B|)^{k-4} .
$$

If $p$ is analytic in $\mathbb{D}$ and $1+\beta\left(z p^{\prime}(z)\right)^{2} / p^{k}(z) \in \mathcal{P}[D, E]$, then $p \in \mathcal{P}[A, B]$.

Proof. By considering the domain $\Omega$ as in Theorem 2.3 and the analytic function

$$
\psi(r, s ; z)=1+\beta s^{2} / r^{k}
$$

where $k$ is non-negative integer, we need to show $\psi \in \Psi[\Omega, A, B]$.

(i) Let $0 \leq k<4$. In view of $(2.4)$, we note that

$$
\psi(r, s ; z)=1+\beta \frac{m^{2}(A-B)^{2} e^{2 i \theta}\left(1+B e^{i \theta}\right)^{k}}{\left(1+B e^{i \theta}\right)^{4}\left(1+A e^{i \theta}\right)^{k}}
$$

so that

$$
\begin{aligned}
& |\chi(\psi(r, s ; z), D, E)|=\left|\begin{array}{r}
\frac{\beta m^{2}(A-B)^{2} e^{2 i \theta}}{(D-E)\left(1+A e^{i \theta}\right)^{k}\left(1+B e^{i \theta}\right)^{4-k}-E \beta m^{2}} \\
(A-B)^{2} e^{2 i \theta}
\end{array}\right| \\
& =\frac{|\beta| m^{2}(A-B)^{2}}{\mid(D-E)\left(1+A e^{i \theta}\right)^{k}\left(1+B e^{i \theta}\right)^{4-k}-E \beta m^{2}} \\
& (A-B)^{2} e^{2 i \theta} \\
& \geq \frac{|\beta| m^{2}(A-B)^{2}}{(D-E)(1+|A|)^{k}(1+|B|)^{4-k}+m^{2}\left|E \beta(A-B)^{2}\right|} \\
& =: \phi(m) \text {. }
\end{aligned}
$$

A calculation shows that $\phi^{\prime}(m)>0$ for $m \geq 1$. Therefore $\phi(m) \geq \phi(1)$. The last inequality simplifies to

$$
|\chi(\psi(r, s ; z), D, E)| \geq 1
$$

whenever the inequality (2.5) holds. As a conclusion it is noted that $\psi(r, s ; z) \notin \Omega$. Thus we get the required subordination. 
(ii) Let $k \geq 4$. Proceeding as in (i), we have

$$
\psi(r, s, t ; z)=1+\frac{\beta m^{2}(A-B)^{2} e^{2 i \theta}\left(1+B e^{i \theta}\right)^{k-4}}{\left(1+A e^{i \theta}\right)^{k}} .
$$

so that

$$
\begin{aligned}
|\chi(\psi(r, s ; z), D, E)| & =\left|\begin{array}{c}
\beta m^{2}(A-B)^{2} e^{2 i \theta}\left(1+B e^{i \theta}\right)^{k-4} \\
(D-E)\left(1+A e^{i \theta}\right)^{k}-E \beta m^{2}(A-B)^{2} \\
\left(1+B e^{i \theta}\right)^{k-4} e^{2 i \theta}
\end{array}\right| \\
& \geq \frac{|\beta| m^{2}(A-B)^{2}\left|\left(1+B e^{i \theta}\right)^{k-4}\right|}{\left|\left(1+A e^{i \theta}\right)^{k}(D-E)\right|+m^{2}\left|E \beta(A-B)^{2} e^{2 i \theta}\right|} \\
& \geq \frac{\left|\left(1+B e^{i \theta}\right)^{k-4}\right|}{(1+|A|)^{k}(D-E)+m^{2}\left|E \beta(A-B)^{2}\right|(1+|B|)^{k-4}} \\
& =: \phi(m) .
\end{aligned}
$$

A calculation shows that $\phi(m)$ is an increasing function for $m \geq 1$ and thus has minimum value at $m=1$. As similar analysis of previous case, we get $p \in \mathcal{P}[A, B]$.

In [18], a lower bound on $\beta$ is determined such that

$$
p(z)+\beta z p^{\prime}(z) / p^{2}(z) \prec \sqrt{1+z}
$$

implies

$$
p(z) \prec \sqrt{1+z} .
$$

Recently, Sharma and Ravichandran [22] established similar type subordination for analytic functions associated to Cardioid. Motivated by this work, the condition on $\beta$ is computed so that $p(z)+\beta z p^{\prime}(z) / p^{2}(z) \in \mathcal{P}[D, E]$ implies $p \in \mathcal{P}[A, B]$.

Theorem 2.6. Suppose $-1 \leq B<A \leq 1$ and $-1 \leq E<D \leq 1$ satisfy

$$
\begin{aligned}
(A-B)\left(|\beta|(1-|B|)-(1+|A|)^{2}\right) & \geq(1+|A|)^{2}((D-E)+|D B-E A|) \\
& +|E \beta(A-B)|(1+|B|) .
\end{aligned}
$$

If $p$ is analytic in $\mathbb{D}$ and $p(z)+\beta z p^{\prime}(z) / p^{2}(z) \in \mathcal{P}[D, E]$, then $p \in \mathcal{P}[A, B]$.

Proof. Consider the domain $\Omega$ as in Theorem 2.3.

The analytic function $\psi: \mathbb{C} \backslash\{0\} \times \mathbb{C} \times \mathbb{D} \rightarrow \mathbb{D}$ is defined as

$$
\psi(r, s ; z)=r+\beta s / r^{2} .
$$

For required subordination, we need to show $\psi(r, s, t, z) \notin \Omega$. For the values of $r, s$ in (2.4), we have

$$
\psi(r, s ; z)=\frac{\left(1+A e^{i \theta}\right)^{3}+\beta m e^{i \theta}(A-B)\left(1+B e^{i \theta}\right)}{\left(1+A e^{i \theta}\right)^{2}\left(1+B e^{i \theta}\right)}
$$


so that

$$
\begin{aligned}
|\chi(\psi(r, s ; z), D, E)| & =\frac{(A-B)\left|\beta m\left(1+B e^{i \theta}\right)+\left(1+A e^{i \theta}\right)^{2}\right|}{\mid\left(1+A e^{i \theta}\right)^{2}\left(D\left(1+B e^{i \theta}\right)-E\left(1+A e^{i \theta}\right)\right)} \\
& =\frac{(A-B)\left|\beta m\left(1+B e^{i \theta}\right)+\left(1+A e^{i \theta}\right)^{2}\right|}{\mid\left(1+A e^{i \theta}\right)^{2}\left((D-E)+(D B-E A) e^{i \theta}\right)} \\
& -E \beta m e^{i \theta}(A-B)\left(1+B e^{i \theta}\right) \mid \\
& \frac{(A-B)\left|\beta m\left(1+B e^{i \theta}\right)\right|-\left|\left(1+A e^{i \theta}\right)^{2}\right|}{\left|\left(1+A e^{i \theta}\right)^{2}\left((D-E)+(D B-E A) e^{i \theta}\right)\right|} \\
& \geq \frac{\left(A \beta m e^{i \theta}(A-B)\left(1+B e^{i \theta}\right) \mid\right.}{(1+|A|)^{2}((D-E)+|D B-E A|)} \\
& =: \phi(m) . \quad+E \beta(A-B) \mid(1+|B|)
\end{aligned}
$$

The function $\phi(m)$ is an increasing for $m \geq 1$. So the function $\phi(m)$ attains its minimum value at $m=1$. Then $|\chi(\psi(r, s ; z), D, E)| \geq \phi(1) \geq 1$ provided the inequality (2.7) holds. By Theorem 2.2, we have $\psi \in \Psi(\Omega ; A, B)$ and this proves the result.

In [25], authors derived condition on $\alpha$ and $\beta$ so that subordination

$$
(1-\alpha) p(z)+\alpha p^{2}(z)+\beta z p^{\prime}(z) / p^{k}(z) \prec 1+\frac{4}{3} z+\frac{2}{3} z^{2} \quad(k=0,1)
$$

implies

$$
p(z) \prec 1+\frac{4}{3} z+\frac{2}{3} z^{2} .
$$

In view of this work, next two theorems give a relation between $\alpha$ and $\beta$ so that

$$
(1-\alpha) p(z)+\alpha p^{2}(z)+\beta z p^{\prime}(z) / p^{k}(z) \in \mathcal{P}[D, E]
$$

(where $k=0,1$ ) implies $p \in \mathcal{P}[A, B]$.

Theorem 2.7. Let $-1 \leq B<A \leq 1,-1 \leq E<0<D \leq 1, \beta \neq 0$ and $0 \leq \alpha \leq 1$. Assume that

$$
\begin{aligned}
(A-B)(|\beta|-(1+|B|)-\alpha(1+|A|)) & \geq(1+|B|)(D(1+|B|) \\
& -E(1-\alpha)(1+|A|))-E \alpha(1+|A|)^{2} \\
& +|E \beta(A-B)| .
\end{aligned}
$$

If $p$ is analytic in $\mathbb{D}$ and $(1-\alpha) p(z)+\alpha p^{2}(z)+\beta z p^{\prime}(z) \in \mathcal{P}[D, E]$, then $p \in \mathcal{P}[A, B]$.

Proof. Consider the domain $\Omega$ as in Theorem 2.3. The analytic function $\psi: \mathbb{C}^{3} \times \mathbb{D} \rightarrow$ $\mathbb{D}$ is defined as

$$
\psi(r, s ; z)=(1-\alpha) r+\alpha r^{2}+\beta s .
$$

To show $\psi \in \Psi[\Omega, A, B]$, it suffices to prove $|\chi(\psi(r, s ; z), D, E)| \geq 1$. It is easy to deduce that

$$
\psi(r, s ; z)=\frac{(1-\alpha)\left(1+A e^{i \theta}\right)\left(1+B e^{i \theta}\right)+\alpha\left(1+A e^{i \theta}\right)^{2}+\beta m(A-B) e^{i \theta}}{\left(1+B e^{i \theta}\right)^{2}}
$$


such that

$$
\begin{aligned}
|\chi(\psi(r, s ; z), D, E)| & =\frac{(A-B)\left|\beta m+\left(1+B e^{i \theta}\right)+\alpha\left(1+A e^{i \theta}\right)\right|}{\mid\left(1+B e^{i \theta}\right)\left(D\left(1+B e^{i \theta}\right)-E(1-\alpha)\left(1+A e^{i \theta}\right)\right)} \\
& \geq \frac{(A-B)(|\beta| m-(1+|B|)-\alpha(1+|A|))}{\left|\left(1+B e^{i \theta}\right)\right|\left|\left(D\left(1+B e^{i \theta}\right)-E(1-\alpha)\left(1+A e^{i \theta}\right)\right)\right|} \\
& \geq \frac{(A-B)(|\beta| m-(1+|B|)-\alpha(1+|A|)}{(1+|B|)(D(1+|B|)-E(1-\alpha)(1+|A|))} \\
& =: \phi(m) . \quad-E \alpha(1+|A|)^{2}+m|E \beta(A-B)|
\end{aligned}
$$

Note that $\phi(m) \geq \phi(1)$ for $m \geq 1$ and therefore $|\chi(\psi(r, s ; z), D, E)| \geq 1$ whenever the inequality $(2.8)$ holds. Thus $\psi(r, s ; z) \notin \Omega$ and Theorem 2.2 yields the desired subordination.

As an implication of Theorems 2.5-2.7, each of following is sufficient condition for function $f \in \mathcal{S}^{*}[A, B]$ :

$$
\text { (a) } \frac{z f^{\prime}(z)}{f(z)}\left(1+\beta\left(\frac{z f^{\prime}(z)}{f(z)}\right)^{-1}\left(1+\frac{z f^{\prime \prime}(z)}{f^{\prime}(z)}-\frac{z f^{\prime}(z)}{f(z)}\right)^{2}\right) \in \mathcal{P}[D, E]
$$

where $-1 \leq B<A \leq 1,-1 \leq E<0<D \leq 1$ and $\beta$ satisfies following inequality

$$
\begin{gathered}
|\beta|(A-B)^{2} \geq(D-E)(1+|A|)^{2}(1+|B|)^{2}+\left|E \beta(A-B)^{2}\right|, \\
\text { (b) } \frac{z f^{\prime}(z)}{f(z)}\left(1+\beta\left(\frac{z f^{\prime}(z)}{f(z)}\right)^{-2}\left(1+\frac{z f^{\prime \prime}(z)}{f^{\prime}(z)}-\frac{z f^{\prime}(z)}{f(z)}\right)\right) \in \mathcal{P}[D, E],
\end{gathered}
$$

where $-1 \leq B<A \leq 1,-1 \leq E<D \leq 1$ and $\beta$ satisfies an inequality (2.7).

(c) $(1-\alpha+\beta) \frac{z f^{\prime}(z)}{f(z)}+(\alpha-\beta)\left(\frac{z f^{\prime}(z)}{f(z)}\right)^{2}+\beta \frac{z^{2} f^{\prime \prime}(z)}{f^{\prime}(z)} \in \mathcal{P}[D, E]$

whenever $\beta \neq 0,-1 \leq B<A \leq 1,-1 \leq E<0<D \leq 1$ and the inequality (2.8) holds.

Corollary 2.8. Let $p \in \mathcal{P}$. For $-1 \leq B<A \leq 1,-1 \leq E<0<D \leq 1$ and $\beta \neq 0$. We assume that

$$
\begin{aligned}
& (A-B)(|\beta|-(1+|B|)) \geq(D-E)+|2 B D-E(A+B)| \\
& +|E \beta(A-B)|+\left|D B^{2}-E A B\right| .
\end{aligned}
$$

If $p(z)+\beta z p^{\prime}(z) \in \mathcal{P}[D, E]$, then $p \in \mathcal{P}[A, B]$.

Corollary 2.9. Let $p \in \mathcal{P}, \beta \neq 0,-1 \leq B<A \leq 1$ and $-1 \leq E<0<D \leq 1$.

We assume the following inequality

$$
\begin{aligned}
(A-B)(|\beta|-(1+|B|)-(1+|A|)) & \geq D(1+|B|)^{2}-E(1+|A|)^{2} \\
& +|E \beta(A-B)| .
\end{aligned}
$$

If $p^{2}(z)+\beta z p^{\prime}(z) \in \mathcal{P}[D, E]$, then $p \in \mathcal{P}[A, B]$. 
Theorem 2.10. Let $p \in \mathcal{P},-1 \leq B<A \leq 1,-1 \leq E<0<D \leq 1, G=1+|A|$ and $0 \leq \alpha \leq 1$. Assume that

$$
\begin{aligned}
& \quad(A-B)\left(|\beta|-G-\alpha(G)^{2}(1-|B|)^{-1}\right) \\
& \quad \geq(G)(D(1+|B|)-E(1-\alpha)(G))-E \alpha(G)^{3}(1-|B|)^{-1}+|E \beta(A-B)| \\
& \text { If }(1-\alpha) p(z)+\alpha p^{2}(z)+\beta z p^{\prime}(z) / p(z) \in \mathcal{P}[D, E], \text { then } p \in \mathcal{P}[A, B] .
\end{aligned}
$$

Proof. By considering $\Omega$ be as in Theorem 2.3 and the analytic function

$$
\psi(r, s, t ; z)=(1-\alpha) r+\alpha r^{2}+\beta s / r,
$$

it is enough to prove $\mid \chi(\psi(r, s ; z) \mid \geq 1$. Using (2.4), we have

$$
\psi(r, s ; z)=\frac{(1-\alpha)\left(1+A e^{i \theta}\right)^{2}\left(1+B e^{i \theta}\right)+\alpha\left(1+A e^{i \theta}\right)^{3}+\beta m(A-B)\left(1+B e^{i \theta}\right) e^{i \theta}}{\left(1+A e^{i \theta}\right)\left(1+B e^{i \theta}\right)^{2}} .
$$

A simple computation yields

$$
\begin{aligned}
& \mid \chi(\psi(r, s ; z) \mid=\left|\begin{array}{c}
\left(1+A e^{i \theta}\right)^{2}\left(1+B e^{i \theta}\right)+\alpha\left(1+A e^{i \theta}\right)^{2}(A-B) e^{i \theta}+ \\
\frac{\beta m(A-B)\left(1+B e^{i \theta}\right) e^{i \theta}-\left(1+A e^{i \theta}\right)\left(1+B e^{i \theta}\right)^{2}}{D\left(1+A e^{i \theta}\right)\left(1+B e^{i \theta}\right)^{2}-E\left((1-\alpha)\left(1+A e^{i \theta}\right)^{2}\right.} \\
\left.\left(1+B e^{i \theta}\right)+\alpha\left(1+A e^{i \theta}\right)^{3}+\beta m(A-B)\left(1+B e^{i \theta}\right) e^{i \theta}\right)
\end{array}\right| \\
&=\frac{(A-B)\left|\beta m+\left(1+A e^{i \theta}\right)+\alpha\left(1+A e^{i \theta}\right)^{2}\left(1+B e^{i \theta}\right)^{-1}\right|}{\mid\left(1+A e^{i \theta}\right)\left(D\left(1+B e^{i \theta}\right)-E(1-\alpha)\left(1+A e^{i \theta}\right)\right)-} \\
& \geq \frac{\left(A-B\left(1+A e^{i \theta}\right)^{3}\left(1+B e^{i \theta}\right)^{-1}-E \beta m e^{i \theta}(A-B) \mid\right.}{\left|\left(1+A e^{i \theta}\right)\left(D\left(1+B e^{i \theta}\right)-E(1-\alpha)\left(1+A e^{i \theta}\right)\right)\right|+} \\
& \geq \frac{(A-B)\left(1+\beta \mid m-(1+|A|)-\alpha(1+|A|)^{2}(1-|B|)^{-1}\right)}{(1+|A|)(D(1+|B|)-E(1-\alpha)(1+|A|))-}=: \phi(m) . \\
& E \alpha(1+|A|)^{3}(1-|B|)^{-1}+m|E \beta(A-B)|
\end{aligned}
$$

It is observed that $\phi^{\prime}(m)>0$ for all $m \geq 1$. As computation done in the previous theorem, we get the required subordination result.

Corollary 2.11. Let $p \in \mathcal{P}, \beta \neq 0,-1 \leq B<A \leq 1$ and $-1 \leq E<0<D \leq 1$. Suppose that

$$
\begin{aligned}
(A-B)(|\beta|-(1+|A|)) \geq(D-E)+|D(B+A)-2 E A| & +|E \beta(A-B)| \\
& +\left|D B A-E A^{2}\right| .
\end{aligned}
$$

If $p(z)+\beta z p^{\prime}(z) / p(z) \in \mathcal{P}[D, E]$, then $p \in \mathcal{P}[A, B]$.

For a positive integer $k$, the condition on $\beta$ is determined so that

$$
(1 / p(z))-\beta z p^{\prime}(z) / p^{k}(z) \in \mathcal{P}[D, E]
$$

implies $p \in \mathcal{P}[A, B]$. 
Theorem 2.12. Let $p \in \mathcal{P},-1 \leq B<A \leq 1$ and $-1 \leq E<0<D \leq 1$. Then $p \in \mathcal{P}[A, B]$ for each of the following subordination conditions:

(a) $(1 / p(z))-\beta z p^{\prime}(z) \in \mathcal{P}[D, E]$ where $\beta$ satisfies

$$
\begin{aligned}
& (A-B)\left(|\beta|(1-|A|)-(1+|B|)^{2}\right) \\
& \geq(1+|B|)^{2}(D(1+|A|)-E(1+|B|))+|E \beta(A-B)|(1+|A|)
\end{aligned}
$$

(b) $(1 / p(z))-\beta z p^{\prime}(z) / p^{k}(z) \in \mathcal{P}[D, E]$ for $k=1,2$ where $\beta$ satisfies

$$
\begin{aligned}
& (A-B)\left(|\beta|-(1+|A|)^{k-1}(1+|B|)^{2-k}\right) \\
& \geq(1+|A|)^{k-1}(1+|B|)^{2-k}(D(1+|A|)-E(1+|B|)) \\
& \quad+|E \beta(A-B)| .
\end{aligned}
$$

(c) $(1 / p(z))-\beta z p^{\prime}(z) / p^{k}(z) \in \mathcal{P}[D, E]$ for $k>2$ where $\beta$ satisfies

$$
\begin{aligned}
& (A-B)\left(|\beta|(1-|B|)^{k-2}-(1+|A|)^{k-1}\right) \\
& \geq(1+|A|)^{k-1}(D(1+|A|)-E(1+|B|)) \\
& \quad+|E \beta(A-B)|(1+|B|)^{k-2} .
\end{aligned}
$$

Proof. For $k=0,1,2,3, \ldots$, let $\Omega$ as in Theorem 2.3 and the function $\psi$ be defined as

$$
\psi(r, s ; z)=\frac{1}{r}-\beta \frac{s}{r^{k}} .
$$

In view of (2.4), the function $\psi$ takes the following shape:

$$
\psi(r, s ; z)=\frac{1+B e^{i \theta}}{1+A e^{i \theta}}-\frac{\beta m e^{i \theta}(A-B)\left(1+B e^{i \theta}\right)^{k}}{\left(1+B e^{i \theta}\right)^{2}\left(1+A e^{i \theta}\right)^{k}} .
$$

(a) For $k=0$, we have

$$
\begin{aligned}
& |\chi(\psi(r, s ; z), D, E)|=\frac{\left|(A-B)\left(\left(1+B e^{i \theta}\right)^{2}+\beta m\left(1+A e^{i \theta}\right)\right)\right|}{\mid\left(1+B e^{i \theta}\right)^{2}\left(D\left(1+A e^{i \theta}\right)-E\left(1+B e^{i \theta}\right)\right)} \\
& +E \beta m(A-B) e^{i \theta}\left(1+A e^{i \theta}\right) \mid \\
& \geq \frac{(A-B)\left(|\beta| m\left|1+A e^{i \theta}\right|-\left|\left(1+B e^{i \theta}\right)^{2}\right|\right)}{\left|\left(1+B e^{i \theta}\right)^{2}\left(D\left(1+A e^{i \theta}\right)-E\left(1+B e^{i \theta}\right)\right)\right|} \\
& +\left|E \beta m(A-B) e^{i \theta}\left(1+A e^{i \theta}\right)\right| \\
& \begin{array}{r}
\geq \frac{(A-B)\left(|\beta| m(1-|A|)-(1+|B|)^{2}\right)}{\left|\left(1+B e^{i \theta}\right)^{2}\right|\left|\left(D\left(1+A e^{i \theta}\right)-E\left(1+B e^{i \theta}\right)\right)\right|} \\
+\left|E \beta m(A-B)\left(1+A e^{i \theta}\right)\right|
\end{array} \\
& \geq \frac{(A-B)\left(|\beta| m(1-|A|)-(1+|B|)^{2}\right)}{(1+|B|)^{2}\left(\left|D\left(1+A e^{i \theta}\right)\right|+\left|E\left(1+B e^{i \theta}\right)\right|\right)} \\
& +|E \beta m(A-B)|\left|\left(1+A e^{i \theta}\right)\right| \\
& \geq \frac{(A-B)\left(|\beta| m(1-|A|)-(1+|B|)^{2}\right)}{(1+|B|)^{2}(D(1+|A|) \mid-E(1+|B|))}=: \phi(m) \text {. } \\
& +m|E \beta(A-B)|(1+|A|)
\end{aligned}
$$


Using first derivative test we note that $\phi$ is an increasing function for $m \geq 1$. Thus the function $\phi(m)$ has minimum value at $m=1$. Therefore $|\chi(\psi(r, s ; z), D, E)| \geq 1$ whenever the inequality (2.12) holds. Thus Theorem 2.2 complete the desired proof. Part (b) and (c) can be proved as part (a). We are omitting further details here.

Let $\beta \neq 0,-1 \leq B<A \leq 1$ and $-1 \leq E<0<D \leq 1$. If one of the following subordination holds for $f \in \mathcal{A}$ :

(i) For $(A-B)(|\beta|-(1+|B|)) \geq(1+|B|)(D(1+|A|)-E(1+|B|))+|E \beta(A-B)|$,

$$
\left(\frac{z f^{\prime}(z)}{f(z)}\right)^{-1}\left(-\beta \frac{z f^{\prime}(z)}{f(z)}\left(1+\frac{z f^{\prime \prime}(z)}{f^{\prime}(z)}-\frac{z f^{\prime}(z)}{f(z)}\right)\right) \in \mathcal{P}[D, E]
$$

(ii) For $(A-B)(|\beta|-(1+|A|)) \geq(1+|A|)(D(1+|A|)-E(1+|B|))+|E \beta(A-B)|$,

$$
\left(\frac{z f^{\prime}(z)}{f(z)}\right)^{-1}\left(1-\beta\left(1+\frac{z f^{\prime \prime}(z)}{f^{\prime}(z)}-\frac{z f^{\prime}(z)}{f(z)}\right)\right) \in \mathcal{P}[D, E]
$$

then $f \in \mathcal{S}^{*}[A, B]$.

Motivated by the work in [2], we obtain the conditions on $A, B, D, E$ for a general Briot-Bouquet differential subordination in the following theorem.

Theorem 2.13. Let $-1 \leq B<A \leq 1,-1 \leq E<D \leq 1$ and $\beta \gamma>0$ satisfy

$$
\begin{aligned}
& (A-B)\left((1-|B|)-(\beta(1+|A|)+\gamma(1+|B|))^{2}\right) \\
& \geq(\beta(1+|A|)+\gamma(1+|B|))^{2}(D-E+|D B-E A|)|E|(A-B)(1+|B|) .
\end{aligned}
$$

If $p \in \mathcal{P}$ satifies the following subordination

$$
p(z)+\frac{z p^{\prime}(z)}{(\beta p(z)+\gamma)^{2}} \in \mathcal{P}[D, E],
$$

then $p \in \mathcal{P}[A, B]$.

Proof. Let $\Omega$ be defined as in Theorem 2.3. Consider the analytic function

$$
\psi(r, s ; z)=r+\frac{s}{(\beta r+\gamma)^{2}}
$$

The required subordination is obtained if we show $\psi \in \Psi[\Omega, A, B]$ by making use of Theorem 2.2. Using (2.4), the function $\psi(r, s ; z)$ takes the following form

$$
\psi(r, s ; z)=\frac{\left(1+A e^{i \theta}\right)\left(\beta\left(1+A e^{i \theta}\right)+\gamma\left(1+B e^{i \theta}\right)\right)^{2}+m(A-B)\left(1+B e^{i \theta}\right) e^{i \theta}}{\left(1+B e^{i \theta}\right)\left(\beta\left(1+A e^{i \theta}\right)+\gamma\left(1+B e^{i \theta}\right)\right)^{2}}
$$


so that

$$
\begin{aligned}
& \mid(A-B)\left(\left(\beta\left(1+A e^{i \theta}\right)+\gamma\left(1+B e^{i \theta}\right)\right)^{2}\right. \\
& |\chi(\psi(r, s ; z), D, E)|=\frac{\left.+m\left(1+B e^{i \theta}\right)\right) \mid}{\mid\left(D\left(1+B e^{i \theta}\right)-E\left(1+A e^{i \theta}\right)\right)\left(\beta\left(1+A e^{i \theta}\right)\right.} \\
& \left.+\gamma\left(1+B e^{i \theta}\right)\right)^{2}-E m e^{i \theta}(A-B)\left(1+B e^{i \theta}\right) \mid \\
& (A-B)\left(m\left|1+B e^{i \theta}\right|-\mid\left(\beta\left(1+A e^{i \theta}\right)\right.\right. \\
& \geq \frac{\left.\left.+\gamma\left(1+B e^{i \theta}\right)\right)^{2} \mid\right)}{\left|(D-E)\left(\beta\left(1+A e^{i \theta}\right)+\gamma\left(1+B e^{i \theta}\right)\right)^{2}\right|} \\
& +\left|e^{i \theta}(D B-E A)\left(\beta\left(1+A e^{i \theta}\right)+\gamma\left(1+B e^{i \theta}\right)\right)^{2}\right| \\
& +\left|E m e^{i \theta}(A-B)\left(1+B e^{i \theta}\right)\right| \\
& (A-B)\left(m(1-|B|)-\left(\left|\beta\left(1+A e^{i \theta}\right)\right|\right.\right. \\
& \geq \frac{\left.\left.+\left|\gamma\left(1+B e^{i \theta}\right)\right|\right)^{2}\right)}{\left|(D-E)\left(\beta\left(1+A e^{i \theta}\right)+\gamma\left(1+B e^{i \theta}\right)\right)^{2}\right|} \\
& +\left|(D B-E A)\left(\beta\left(1+A e^{i \theta}\right)+\gamma\left(1+B e^{i \theta}\right)\right)^{2}\right| \\
& +\left|\operatorname{Em}(A-B)\left(1+B e^{i \theta}\right)\right| \\
& \geq \frac{(A-B)\left(m(1-|B|)-(\beta(1+|A|)+\gamma(1+|B|))^{2}\right)}{(D-E)(\beta(1+|A|)+\gamma(1+|B|))^{2}} \\
& +\left|(D B-E A)(\beta(1+|A|)+\gamma(1+|B|))^{2}\right| \\
& +m|E(A-B)|(1+|B|) \\
& =: \phi(m) \text {. }
\end{aligned}
$$

A computation shows that $\phi^{\prime}(m)>0$. Thus for $m \geq 1, \phi(m) \geq \phi(1)$ and therefore $|\chi(\psi(r, s ; z), D, E)| \geq 1$ whenever the inequality $(2.16)$ holds. This implies that $\psi(r, s ; z) \notin \Omega$. Hence the desired subordination is obtained.

Acknowledgements. The authors are thankful to the referee for pointing out a few typographic errors in the earlier version of the manuscript.

\section{References}

[1] Ahuja, O.P., Kumar, S., Ravichandran, V., Applications of first order differential subordination for functions with positive real part, Stud. Univ. Babeş-Bolyai Math., 63(2018), no. 3, 303-311.

[2] Ali, R.M., Ravichandran, V., Seenivasagan, N., On Bernardi's integral operator and the Briot-Bouquet differential subordination, J. Math. Anal. Appl., 324(2006), 663-668.

[3] Ali, R.M., Ravichandran, V., Seenivasagan, N., Sufficient conditions for Janowski starlikeness, Int. J. Math. Math. Sci., 2007, Art. ID 62925, 7 pp.

[4] Anand, S., Kumar, S., Ravichandran, V., First-order differential subordinations for Janowski starlikeness, in "Mathematical Analysis. I. Approximation Theory", 185-196, Springer Proc. Math. Stat., 306, Springer, Singapore, 2020.

[5] Bohra, N., Kumar, S., Ravichandran, V., Some special differential subordinations, Hacet. J. Math. Stat., 48(2019), no. 4, 1017-1034.

[6] Bulboacă, T., Differential Subordinations and Superordinations, Recent Results, House of Scientific Book Publ., Cluj-Napoca, 2005. 
[7] Cho, N.E., Kumar, S., Kumar, V., Ravichandran, V., Differential subordination and radius estimates for starlike functions associated with the Booth lemniscate, Turkish J. Math., 42(2018), no. 3, 1380-1399.

[8] Cho, N.E., Kumar, S., Kumar, V., Ravichandran, V., Srivastava, H.M., Starlike functions related to the Bell numbers, Symmetry, 11(2019), no. 2, Article 219, 17 pp.

[9] Chojnacka, O., Lecko, A., Differential subordination of a harmonic mean to a linear function, Rocky Mountain J. Math., 48(2018), no. 5, 1475-1484.

[10] Gandhi, S., Kumar, S., Ravichandran, V., First order differential subordinations for Caratheodory functions, Kyungpook Math. J., 58(2018), 257-270.

[11] Goodman, A.W., Univalent Functions, Vol. II, Mariner Publishing Co., Inc., Tampa, FL, 1983.

[12] Janowski, W., Extremal problems for a family of functions with positive real part and for some related families, Ann. Polon. Math., 23(1970/1971), 159-177.

[13] Kanas, S., Techniques of the differential subordination for domains bounded by conic sections, Int. J. Math. Math. Sci., 2003, no. 38, 2389-2400.

[14] Kanas, S., Differential subordination related to conic sections, J. Math. Anal. Appl., 317(2006), no. 2, 650-658.

[15] Kanas, S., Lecko, A., Differential subordination for domains bounded by hyperbolas, Zeszyty Nauk. Politech. Rzeszowskiej Mat., 175(1999), no. 23, 61-70.

[16] Kim, I.H., Sim, Y.J., Cho, N.E., New criteria for Carathéodory functions, J. Inequal. Appl., 2019, 2019:13.

[17] Kumar, S., Ravichandran, V., Subordinations for functions with positive real part, Complex Anal. Oper. Theory, 12(2018), no. 5, 1179-1191.

[18] Kumar, S.S., Kumar, V., Ravichandran, V., Cho, N.E., Sufficient conditions for starlike functions associated with the lemniscate of Bernoulli, J. Inequal. Appl. 2013(2013), 176, $13 \mathrm{pp}$.

[19] Miller, S.S., Mocanu, P.T., On some classes of first-order differential subordinations, Michigan Math. J., 32(1985), no. 2, 185-195.

[20] Miller, S.S., Mocanu, P.T., Differential Subordinations, Monographs and Textbooks in Pure and Applied Mathematics, 225, Marcel Dekker, Inc., New York, 2000.

[21] Nunokawa, M., Obradović, M., Owa, S., One criterion for univalency, Proc. Amer. Math. Soc., 106(1989), no. 4, 1035-1037.

[22] Ravichandran, V., Sharma, K., Sufficient conditions for starlikeness, J. Korean Math. Soc., 52(2015), no. 4, 727-749.

[23] Robertson, M.S., Certain classes of starlike functions, Michigan Math. J., 32(1985), no. 2, 135-140.

[24] Seoudy, T.M., Aouf, M.K., Classes of admissible functions associated with certain integral operators applied to meromorphic functions, Bull. Iranian Math. Soc., 41(2015), no. $4,793-804$.

[25] Sharma, K., Ravichandran, V., Applications of subordination theory to starlike functions, Bull. Iranian Math. Soc., 42(2016), no. 3, 761-777.

[26] Tuneski, N., Bulboacă, T., Sufficient conditions for bounded turning of analytic functions, Ukraïn. Mat. Zh., 70(2018), no. 8, 1118-1127. 
Swati Anand

Rajdhani College, University of Delhi,

Delhi-110015, India

e-mail: swati_anand01@yahoo.com

Sushil Kumar

Bharati Vidyapeeth's college of Engineering,

Delhi-110063, India

e-mail: sushilkumar16n@gmail.com

V. Ravichandran

Department of Mathematics,

National Institute of Technology,

Tiruchirappalli-620015, India

e-mail: ravic@nitt.edu; vravi68@gmail.com 